\title{
Modellreduktion für hochviskose, nicht-isotherme Fluide mit freier Oberfläche
}

\author{
Edmond Skeli ${ }^{1}$, Dirk Weidemann ${ }^{1}$, Klaus Panreck ${ }^{1}$, \\ ${ }^{1}$ Institut für Systemdynamik und Mechatronik, FH Bielefeld, Interaktion 1, 33619 Bielefeld; \\ \{edmond.skeli,dirk.weidemann,klaus.panreck\}@fh-bielefeld.de
}

Kurzfassung. Mit dem Ziel effiziente Steuerungs-, Regelungs- und/oder Diagnoseverfahren nutzen zu können, greift man auch in der verfahrenstechnischen Industrie vermehrt auf mathematische Modelle, die die zugrundeliegenden physikalischen Prozesse beschreiben, zurück. Hierfür ist es notwendig, mathematische Modelle zu bestimmen, die einerseits hinreichend präzise sind, andererseits aber einen nicht zu hohen Rechenaufwand erfordern. Vor diesem Hintergrund wird die Reduzierung eines Modells, welches das Verhalten eines hochviskosen, nicht-isotherme Fluids mit einer freien Oberfläche beschreibt, erörtert. Das Verhalten des Fluids genügt einem Modell, das aus einem System partieller Differentialgleichungen besteht und neben den zweidimensionalen Navier-Stokes Gleichungen auch die thermische Energiegleichung, die das Temperaturverhalten beschreibt, umfasst. Mit Hilfe der Störungstheorie kann gezeigt werden, dass das Verhalten der Geschwindigkeit und der Temperatur des Fluids durch zwei einfachere Teilmodelle beschrieben werden kann. Das erste Teilmodell dient zur Berechnung der Strömungsdynamik, während das zweite Teilmodell die Berechnung des thermischen Verhaltens ermöglicht.

\section{Einleitung}

In vorliegenden Beitrag wird das Einlaufen eines hochviskosen, nicht-isothermen Fluids in den Spalt zwischen zwei gegenläufig rotierenden Zylindern betrachtet. Hierbei gilt es zu berücksichtigen, dass zum einen der Spalt zu Beginn leer ist und sich erst im Laufe der Zeit mit dem Fluid füllt, sodass das Einlaufen des Fluids als instationäres Prozessverhalten zu beschreiben ist. Zum anderen entsteht während des Füllens vor dem Spalte ein Wulst, dessen Größe sich über der Zeit solange verändert, bis ein stationärer Arbeitspunkt erreicht ist. Da die zeitliche Entwicklung der Wulst und damit die zeitliche Veränderung der Fluidgrenze a-priori unbekannt ist, bedarf es hinsichtlich der Bestimmung des Fluidverhaltens nicht nur der numerischen Lösung eines Systems von partiellen Differentialgleichungen, das aus den inkompressiblen Navier-Stokes Gleichungen und der thermischen Energiegleichung besteht, son- dern auch der Berechnung der Fluidgrenze. Im Weiteren wird die Fluidgrenze, d.h. die an die umgebende Luft angrenzende Oberfläche des Fluids auch als freie Oberfläche bezeichnet.

Einen geeigneten Ansatz zur Berechnung der instationären, inkompressiblen Navier-Stokes-Gleichungen mit freier Oberfläche bietet die Marker- und Cell (MAC)-Methode, die von Harlow und Welch in [1] eingeführt wurde. Amsden und Harlow vereinfachten die MAC-Methode in [2], indem sie die Geschwindigkeitsund Druckberechnungen entkoppelten. Darüber hinaus wird in [3, 4] die MAC-Methode für drei räumliche Dimensionen angepasst. Auf der Grundlage der MACMethode wird in [5] ein Ansatz zur Bestimmung der freien Oberfläche eines hochviskosen, nicht-isothermen Fluids, das in den Spalt zwischen zwei gegensinnig rotierenden Zylindern eintritt, vorgeschlagen. Dieser Ansatz erlaubt zwar die Simulation des instationären Verhaltens des Fluids, hat allerdings den Nachteil, dass er einen hohen Rechenaufwand erfordert, sodass der Ansatz nicht für modellbasierte Steuerungs-, Regelungsund/oder Diagnosezwecke verwendet werden kann.

Um den Rechenaufwand zu verringert, erweist es sich als sinnvoll, das mathematische Modell geeignet zu reduzieren. Hinsichtlich der Charakterisierung des zeitlichen Verhaltens werden daher die partiellen Differentialgleichungen normiert, sodass die einzelnen Zeitkonstanten bestimmt werden können. Diese Zeitkonstanten erlauben nach $[6,7]$ eine qualitative Beurteilung des transienten Verhaltens der physikalischen Größen, d.h. der Geschwindigkeiten und der Temperatur. Mit Hilfe der Störungstheorie (vgl. $[8,9])$ lässt sich zeigen, dass sich die Geschwindigkeiten und die Temperatur des Fluids auf verschiedenen Zeitskalen entwickeln, was darauf hinweist, dass zwei reduzierte Modelle, d.h. ein schnelles und ein langsames Teilmodell verwendet werden können. Mit Hilfe des schnellen Teilmodells erfolgt die Berechnung der Geschwindigkeiten des Fluids, wobei davon ausgegangen wird, dass sich die Fluidtem- 
peratur während dieser Berechnung nicht verändert. Demgegenüber wird mit dem langsamen Teilmodell die Temperatur berechnet.

Im Weiteren werden zunächst das mathematische Fluidmodell in normalisierter Form sowie die schnellen und langsamen Teilmodelle, die mit Hilfe der Störungstheorie bestimmt werden, eingeführt. Im Anschluss erfolgt eine kurze Darstellung der räumliche Diskretisierung der Modellgleichungen und der Marker- und Cell (MAC)-Methode zur Bestimmung der freien Oberfläche. Abschließend werden die numerischen Simulationsergebnisse, die mit Hilfe des reduzierten Modells berechnet worden sind, präsentiert und mit den Ergebnissen des Vollmodells (s. [5]) verglichen.

\section{Normalisierung der Modellgleichungen}

Das zeit- und örtliche Verhalten der Geschwindigkeiten und des Drucks eines hochviskosen, nicht-isothermen Fluids genügt den inkompressiblen Navier-Stokes Gleichungen

$$
\begin{aligned}
\frac{\partial u}{\partial t}+u \frac{\partial u}{\partial x}+v \frac{\partial u}{\partial y} & =-\frac{1}{\rho} \frac{\partial p}{\partial x}+\frac{\eta}{\rho}\left(\frac{\partial^{2} u}{\partial x^{2}}+\frac{\partial^{2} u}{\partial y^{2}}\right) \\
\frac{\partial v}{\partial t}+u \frac{\partial v}{\partial x}+v \frac{\partial v}{\partial y} & =-\frac{1}{\rho} \frac{\partial p}{\partial y}+\frac{\eta}{\rho}\left(\frac{\partial^{2} v}{\partial x^{2}}+\frac{\partial^{2} v}{\partial y^{2}}\right) \\
0 & =\frac{\partial u}{\partial x}+\frac{\partial v}{\partial y}
\end{aligned}
$$

mit den Anfangs- und Randbedingungen

$$
\begin{aligned}
\mathbf{u}(\zeta, 0)=\mathbf{u}_{0}(\zeta) & \forall \zeta \in \Gamma, \\
\mathbf{u}(\zeta, t)=\mathbf{h}(\zeta, t) & \forall(\zeta, t) \in \partial \Gamma \times\left[0, t_{e}\right]
\end{aligned}
$$

wobei $u, v$ Geschwindigkeiten in $x$-, $y$-Richtung repräsentieren. Im Weiteren kennzeichnet $\mathbf{u}=(u, v)^{T}$ : $\Gamma \times \mathbb{R}_{(+)} \rightarrow \mathbb{R}^{2}$ den Vektor der Fluidgeschwindigkeiten, $\mathbf{u}_{0}(\zeta) \in \mathbb{R}^{2}$ die Anfangsbedingungen und $\mathbf{h}: \partial \Gamma \times$ $\mathbb{R}_{(+)} \rightarrow \mathbb{R}^{2}$ die Randbedingungen, wobei $\Gamma \subset \mathbb{R}^{2}$ die Definitionsmenge und $\partial \Gamma$ den Rand der Definitionsmenge darstellt. Ferner ist $p: \Gamma \times \mathbb{R}_{(+)} \rightarrow \mathbb{R}$ der Druck, $\rho \in \mathbb{R}$ die Dichte und $\eta(T) \in \mathbb{R}$ die Viskosität.

Des Weiteren genügt die zeit- und örtliche Entwicklung der Fluidtemperatur der partiellen DGL

$$
\rho C_{p}\left(\frac{\partial T}{\partial t}+u \frac{\partial T}{\partial x}+v \frac{\partial T}{\partial y}\right)=\lambda\left(\frac{\partial^{2} T}{\partial x^{2}}+\frac{\partial^{2} T}{\partial y^{2}}\right)
$$

$$
+2 \eta\left(\frac{\partial u}{\partial x}\right)^{2}+\eta\left(\frac{\partial u}{\partial y}+\frac{\partial v}{\partial x}\right)^{2}+2 \eta\left(\frac{\partial v}{\partial y}\right)^{2},
$$

wobei $T: \Gamma \times \mathbb{R}_{(+)} \rightarrow \mathbb{R}$ die Temperatur darstellt und $C_{p}, \lambda \in \mathbb{R}$ spezifische Wärmekapazität und Wärmeleitfähigkeit sind. Die zugehörigen Anfangs- und Randbedingungen seien duch

$$
\begin{aligned}
T(\zeta, 0) & =T_{0}(\zeta) & & \forall \zeta \in \Gamma, \\
T(\zeta, t) & =d(\zeta, t) & & \forall(\zeta, t) \in \partial \Gamma \times\left[0, t_{e}\right]
\end{aligned}
$$

mit $T_{0}(\zeta) \in \mathbb{R}$ gegeben.

Normalisierung der Variablen ergibt

$$
\begin{aligned}
& u_{n}=\frac{u}{\bar{u}}, \quad v_{n}=\frac{v}{\bar{u}}, \quad \Pi_{p x}=\frac{h^{2}}{\bar{u} \eta} \frac{\partial p}{\partial x}, \\
& \Pi_{p y}=\frac{h^{2}}{\bar{u} \eta} \frac{\partial p}{\partial y}, \quad x_{n}=\frac{x}{L}, \quad y_{n}=\frac{y}{h}, \\
& t_{n}=\frac{t}{\bar{t}}, \quad T_{n}=\frac{T}{\bar{T}}, \quad \eta_{n}=\frac{\eta}{\bar{\eta}},
\end{aligned}
$$

wobei die Größen $u_{n}, v_{n}, \Pi_{n x}, \Pi_{n y}, x_{n}, y_{n}$ die normalisierten Geschwindigkeiten, Druckgradienten, Koordinaten und $t_{n}, T_{n}, \eta_{n}$ die normalisierte Zeit, Temperatur und Viskosität repräsentieren.

Unter Verwendung der normalisierten Variablen aus (5) lässt sich das System aus partiellen Differentialgleichung, welches sowohl die Navier-Stokes Gleichungen als auch die thermische Energiegleichung umfasst, in die Form

$$
\begin{aligned}
\frac{t_{\eta}}{\bar{t}} \frac{\partial u_{n}}{\partial t_{n}} & =h_{1}\left(t_{n}, \mathbf{r}_{n}, \mathbf{u}_{n}, p, T_{n}\right), \\
\frac{t_{\eta}}{\bar{t}} \frac{\partial v_{n}}{\partial t_{n}} & =h_{2}\left(t_{n}, \mathbf{r}_{n}, \mathbf{u}_{n}, p, T_{n}\right), \\
0 & =h_{\nabla}\left(\mathbf{r}_{n}, \mathbf{u}_{n}\right), \\
\frac{\tau_{\lambda}}{\bar{t}} \frac{\partial T_{n}}{\partial t_{n}} & =h_{3}\left(t_{n}, \mathbf{r}_{n}, \mathbf{u}_{n}, p, T_{n}\right)
\end{aligned}
$$

$\operatorname{mit} \mathbf{r}_{n}=\left[x_{n}, y_{n}\right]^{T}, \mathbf{u}_{n}=\left[u_{n}, v_{n}\right]^{T}$ und

$$
\begin{array}{r}
h_{1}\left(t_{n}, \mathbf{r}_{n}, \mathbf{u}_{n}, p, T_{n}\right)=-\operatorname{Re}\left(\frac{h}{L} u_{n} \frac{\partial u_{n}}{\partial x_{n}}+v_{n} \frac{\partial u_{n}}{\partial y_{n}}\right) \\
-\Pi_{p x}+\eta_{n}\left(\left(\frac{h}{L}\right)^{2} \frac{\partial^{2} u_{n}}{\partial x_{n}^{2}}+\frac{\partial^{2} u_{n}}{\partial y_{n}^{2}}\right), \\
h_{2}\left(t_{n}, \mathbf{r}_{n}, \mathbf{u}_{n}, p, T_{n}\right)=-\operatorname{Re}\left(\frac{h}{L} u_{n} \frac{\partial v_{n}}{\partial x_{n}}+v_{n} \frac{\partial v_{n}}{\partial y_{n}}\right)
\end{array}
$$




$$
\begin{array}{r}
-\Pi_{p y}+\eta_{n}\left(\left(\frac{h}{L}\right)^{2} \frac{\partial^{2} v_{n}}{\partial x_{n}^{2}}+\frac{\partial^{2} v_{n}}{\partial y_{n}^{2}}\right) \\
h_{\nabla}\left(\mathbf{r}_{n}, \mathbf{u}_{n}\right)=\frac{\bar{u}}{h}\left(\frac{h}{L} \frac{\partial u_{n}}{\partial x_{n}}+\frac{\partial v_{n}}{\partial y_{n}}\right), \\
h_{3}\left(t_{n}, \mathbf{r}_{n}, \mathbf{u}_{n}, p, T_{n}\right)=-G r\left(u_{n} \frac{\partial T_{n}}{\partial x_{n}}+v_{n} \frac{L}{h} \frac{\partial T_{n}}{\partial y_{n}}\right) \\
+\left(\left(\frac{h}{L}\right)^{2} \frac{\partial^{2} T_{n}}{\partial x_{n}^{2}}+\frac{\partial^{2} T_{n}}{\partial y_{n}^{2}}\right)+2 \eta_{n} B r\left(\frac{h}{L}\right)^{2}\left(\frac{\partial u_{n}}{\partial x_{n}}\right)^{2} \\
+\eta_{n} B r\left(\frac{\partial u_{n}}{\partial y_{n}}+\frac{h}{L} \frac{\partial v_{n}}{\partial x_{n}}\right)^{2}+2 \eta_{n} B r\left(\frac{\partial v_{n}}{\partial y_{n}}\right)^{2}
\end{array}
$$

überführen. Hierbei stellen

$$
R e=\frac{\rho h \bar{u}}{\bar{\eta}}, \quad B r=\frac{\bar{\eta} \bar{u}^{2}}{\lambda \bar{T}}, \quad G r=\frac{\bar{u} h^{2}}{a L}
$$

die Reynolds-, Brinkmann-, and Graetz-Zahlen und

$$
t_{\eta}=\frac{\rho h^{2}}{\bar{\eta}}, \quad \tau_{\lambda}=\frac{h^{2} \rho C_{p}}{\lambda}
$$

die viskose Relaxitionszeit bzw. die konduktive thermische Ausgleichszeit dar.

\section{Modellreduktion}

Im Folgenden wird das Verhältnis der beiden in (11) gegebenen Zeitkonstanten näher betrachtet, da diese Aufschluss über das transiente Verhalten der Fluidgeschwindigkeiten und -temperatur geben. Ein Vergleich der beiden Zeitkonstanten

$$
\frac{t_{\eta}}{\tau_{\lambda}}=\frac{\frac{\rho h^{2}}{\bar{\eta}}}{\frac{h^{2} \rho C_{p}}{\lambda}}=\frac{\lambda}{\bar{\eta} C_{p}}
$$

zeigt, dass in Folge der hohen Viskosität

$$
\tau_{\lambda}>>t_{\eta}
$$

gilt. Physikalisch gesehen zeigt (13), dass das transiente Verhalten der Geschwindigkeiten sehr viel schneller ist als das der Temperatur.

Da entweder $\bar{t}=t_{\eta}$ oder $\bar{t}=\tau_{\lambda}$ als Normalisierungskonstante verwendet werden kann, wird in den beiden folgenden Unterabschnitten diskutiert, welchen Effekt die Wahl von $\bar{t}=t_{\eta}$ bzw. $\bar{t}=\tau_{\lambda}$ auf die Gleichungen (6)-(9) hat.

\subsection{Viskose Relaxtionszeit als Normalisierungskonstante}

Wählt man $t_{\eta}$ als Normalisierungskonstante, folgt für die Faktoren auf der linken Seite der Gleichungen (6), (7) und (9) unmittelbar $t_{\eta} / \bar{t}=t_{\eta} / t_{\eta}=1$ und $\tau_{\lambda} / \bar{t}=$ $\tau_{\lambda} / t_{\eta}$, sodass die normalisierten Navier-Stokes Gleichungen die Form

$$
\begin{aligned}
\frac{\partial u_{n}}{\partial t_{n}} & =h_{1}\left(t_{n}, \mathbf{r}_{n}, \mathbf{u}_{n}, p, T_{n}\right), \\
\frac{\partial v_{n}}{\partial t_{n}} & =h_{2}\left(t_{n}, \mathbf{r}_{n}, \mathbf{u}_{n}, p, T_{n}\right), \\
0 & =h_{\nabla}\left(\mathbf{r}_{n}, \mathbf{u}_{n}\right)
\end{aligned}
$$

annehmen. Wählt man darüber hinaus $t_{\eta} / \tau_{\lambda}=\varepsilon$ mit $\varepsilon<<1$ als Störungsparameter und multipliziert die thermische Energiegleichung (9) mit $\varepsilon$ ergibt sich

$$
\frac{\partial T_{n}}{\partial t_{n}}=\varepsilon h_{3}\left(t_{n}, \mathbf{r}_{n}, \mathbf{u}_{n}, p, T_{n}\right)=0 .
$$

Das durch die Gleichungen (14)-(16) beschriebene Modell wird als schnelles Teilmodell bezeichnet. Es wird zur Berechnung der Geschwindigkeiten bei Impulsänderungen verwendet. Da sich die Temperatur über $t_{\eta}$ nicht signifikant ändert, muss die partielle Differentialgleichung (9) nicht gelöst werden. Vielmehr kann die Temperatur, wie in (17) angegeben, als konstante Größe angenommen werden.

\subsection{Konduktive thermische Ausgleichszeit als Normalisierungskonstante}

Im Unterschied zu der in Abschnitt 2.1 beschriebenen Vorgehensweise führt die Wahl von $\tau_{\lambda}$ als Normalisierungskonstante zu einem System

$$
\begin{aligned}
\varepsilon \frac{\partial u_{n}}{\partial t_{n}} & =h_{1}\left(t_{n}, \mathbf{r}_{n}, \mathbf{u}_{n}, p, T_{n}\right), \\
\varepsilon \frac{\partial v_{n}}{\partial t_{n}} & =h_{2}\left(t_{n}, \mathbf{r}_{n}, \mathbf{u}_{n}, p, T_{n}\right), \\
0 & =h_{\nabla}\left(\mathbf{r}_{n}, \mathbf{u}_{n}\right), \\
\frac{\partial T_{n}}{\partial t_{n}} & =h_{3}\left(t_{n}, \mathbf{r}_{n}, \mathbf{u}_{n}, p, T_{n}\right)
\end{aligned}
$$

von singulär gestörten partiellen Differentialgleichungen, wobei $\varepsilon$ wie oben beschrieben definiert ist. Nimmt man an, dass $\varepsilon \rightarrow 0$ gilt, gehen die instationären Navier- 
Stokes Gleichungen in die stationäre Form

$$
\begin{aligned}
& 0=h_{1}\left(t_{n}, \mathbf{r}_{n}, \mathbf{u}_{n}, p, T_{n}\right), \\
& 0=h_{2}\left(t_{n}, \mathbf{r}_{n}, \mathbf{u}_{n}, p, T_{n}\right), \\
& 0=h_{\nabla}\left(\mathbf{r}_{n}, \mathbf{u}_{n}\right) .
\end{aligned}
$$

über.

Das durch (21)-(24) gegebene Modell wird als langsames Teilmodell bezeichnet und dient der Berechnung der Fluidtemperatur. Während dieser Berechnung sind die Geschwindigkeiten nicht als differentielle, sondern vielmehr als algebraische Zustände $\mathrm{zu}$ interpretieren, die so angepasst werden müssen, dass die algebraischen Bedingungen (22)-(24) erfüllt sind.

\subsection{Hybrides Modell}

Im Gegensatz zum Ansatz in [5], bei dem das Verhalten des in den Spalt einlaufenden Fluids mit Hilfe des vollständigen Modells, d.h. mit Hilfe des aus den partiellen Differentialgleichungen (1)-(4) bestehenden Systems bestimmt wird, basiert der in diesem Beitrag vorgestellte Ansatz darauf, das Verhalten mit Hilfe der reduzierten Modellgleichungen (14)-(16) und (21)-(24) $\mathrm{zu}$ berechnen. Es zeigt sich, dass sich die Rechenzeit durch Verwendung der reduzierten Modelle signifikant reduzieren lässt.

Solange keine Impulsänderungen auftritt, erfolgt die Nutzung des langsamen Teilmodells. Ein Impulsänderung tritt auf, wenn das Fluid in eine Zelle des Diskretisierungsgitters (vgl. Abschnitt 3) eintritt, die zum einen noch nicht mit Fluid gefüllt war und zum anderen mit einem der beiden Zylinder in Kontakt steht. Unmittelbar nach dem Erkennen einer Impulsänderung wird das schnelle Teilmodell verwendet. Das schnelle Teilmodell wird so lange genutzt, bis sich die Anzahl der belegten Zellen nicht verändert und die Geschwindigkeiten des Fluids in diesen Zellen stationär sind. Interpretiert man eine Impulsänderung als Ereignis $e_{\mathrm{im}}$ und das Auftreten der lokal stationären Geschwindigkeiten als Ereignis $e_{\text {st }}$, lässt sich das Verhalten des Fluids durch den in Abb. 1 dargestellten hybriden Automaten modellieren.

\section{Ortsdiskretisierung}

Hinsichtlich des numerischen Lösens der partiellen Differentialgleichungen ist eine geeignete räumliche Diskretisierung erforderlich. Wie in Abb. 2 dargestellt, werden die Geschwindigkeiten in der Mitte der verti-

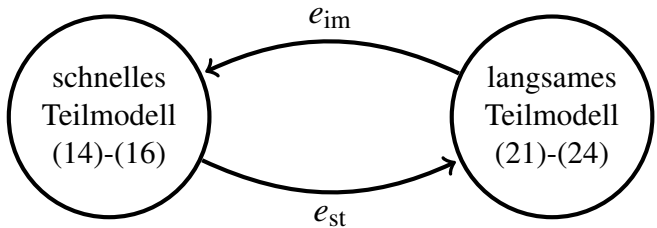

Abb. 1: Reduziertes Modell als hybrider Automat

kalen und horizontalen Kanten des Diskretisierungsgitters berechnet. Im Gegensatz dazu werden sowohl der Druck als auch die Temperatur in der Zellmitte des Gitters berechnet. Eine derartiges Gitter wird auch als staggered grid bezeichnet.

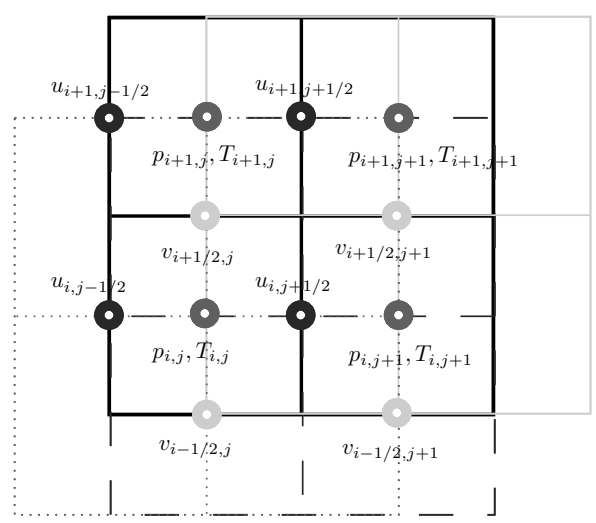

Abb. 2: Diskretisierungsgitter mit Berechnungsknoten

Die partiellen Ableitungen erster Ordnung können entsprechend

$$
\begin{aligned}
D^{x} f_{i, j} & =\frac{f_{i, j+1}-f_{i, j-1}}{2 \Delta x}, \quad D_{-}^{x} f_{i, j}=\frac{f_{i, j}-f_{i, j-1}}{\Delta x}, \\
D_{+}^{x} f_{i, j} & =\frac{f_{i, j+1}-f_{i, j}}{\Delta x}
\end{aligned}
$$

durch Vorwärts-, Rückwärts- und ZentraleDifferenzenquotienten und partielle Ableitungen zweiter Ordnung durch

$$
K^{x} f_{i, j}=\frac{f_{i, j+1}-2 f_{i, j}+f_{i, j-1}}{(\Delta x)^{2}}
$$

mit $i=1,2, \ldots, n_{1}$ und $j=1,2, \ldots, n_{2}$ approximierte werden, wobei $f$ wahlweise $u, v, p$ oder $T$ repräsentiert, $n_{1}, n_{2} \in \mathbb{R}$ die Anzahl der Diskretisierungspunkte in $x$ - und $y$-Richtung darstellen und $\Delta x$ die Schrittweite der Diskretisierung in $x$-Richtung. Die Operati- 
on $D^{y}, D_{-}^{y}, D_{+}^{y}$ und $K^{y}$ sind in analoger Weise definiert, woebi $\Delta y=f_{\Delta y}(x)$ die variable Diskretisierungsschrittweite in $y$-Richtung ist, vgl. [5]. Es sei angemerkt, dass in diesem und in Abschnitt 5 infolge der Ortsdiskretisierung $\mathbf{u}: \Gamma \times \mathbb{R}_{(+)} \rightarrow \mathbb{R}^{2\left(n_{1}+n_{2}\right)}, p: \Gamma \times \mathbb{R}_{(+)} \rightarrow \mathbb{R}^{\left(n_{1}+n_{2}\right)}$ und $T: \Gamma \times \mathbb{R}_{(+)} \rightarrow \mathbb{R}^{\left(n_{1}+n_{2}\right)}$ gilt.

Die Diskretisierung überführt das schnelle Teilsystem (14)-(16) in die Form

$$
\begin{aligned}
I \dot{\mathbf{u}}(t) & =K(\mathbf{u}) \mathbf{u}(t)-B p(t)+\mathbf{f}(\mathbf{u}(t), p(t)), \\
0 & =B^{T} \mathbf{u}(t)
\end{aligned}
$$

und das langsame Teilsystem (21)-(24) in die Form

$$
\begin{aligned}
0 & =K(\mathbf{u}) \mathbf{u}(t)-B p(t)+\mathbf{f}(\mathbf{u}(t), p(t)), \\
0 & =B^{T} \mathbf{u}(t), \\
I \dot{T}(t) & =K_{T}(\mathbf{u}) T(t)+D(\mathbf{u})+g(T(t))
\end{aligned}
$$

mit $B=\left[D_{+}^{x}, D_{+}^{y}\right]^{T}$. Hierbei stellt $I$ die Einheitsmatrix dar und

$$
K(\mathbf{u})=\left[\begin{array}{cc}
K_{1}+N_{1}(\mathbf{u}) & 0 \\
0 & K_{2}+N_{1}(\mathbf{u})
\end{array}\right]
$$

mit $K_{1}=K_{2}=K^{x}+K^{y}$ repräsentiert den linearen Diffusionsterm und

$$
N(\mathbf{u})=\left[\begin{array}{l}
N_{1}(\mathbf{u}) \\
N_{2}(\mathbf{u})
\end{array}\right]=\left[\begin{array}{l}
u_{i, j+1 / 2} D^{x}+v_{i, j+1 / 2}^{*} D^{y} \\
u_{i+1 / 2, j}^{*} D^{x}+v_{i+1 / 2, j} D^{y}
\end{array}\right]
$$

den nichtlinearen Konvektionsterm mit

$$
\begin{aligned}
& u_{i+1 / 2, j}^{*}= \frac{1}{4}\left(u_{i, j-1 / 2}+u_{i, j+1 / 2}+\right. \\
&\left.u_{i+1, j+1 / 2}+u_{i+1, j-1 / 2}\right), \\
& v_{i, j+1 / 2}^{*}=\frac{1}{4}\left(v_{i-1 / 2, j}+v_{i+1 / 2, j}+\right. \\
&\left.v_{i+1 / 2, j+1}+v_{i-1 / 2, j+1}\right) .
\end{aligned}
$$

Es gilt zu berücksichtigen, dass $\mathbf{f}(\mathbf{u}(t), p(t))$ und $g(T(t))$ von den zeitveränderlichen Randbedingungen abhängen, sodass diese Funktionen entsprechend der freien Oberfläche (vgl. Abschnitt 4) anzupassen sind. Ferner sind die Operatoren für die Berechnung der Temperatur durch

$$
D(\mathbf{u})=2 \eta\left(\left(D_{+}^{x} u\right)^{2}+\frac{1}{\eta}\left(D_{+}^{y} u+D_{+}^{x} v\right)^{2}+\left(D_{+}^{y} v\right)^{2}\right) .
$$

und $K_{T}(\mathbf{u})=\left(K_{1}+N_{T}(\mathbf{u})\right)$ mit

$$
N_{T}(\mathbf{u})=\frac{u_{i, j-1 / 2}+u_{i, j+1 / 2}}{2} D^{x}+\frac{v_{i-1 / 2, j}+v_{i+1 / 2, j}}{2} D^{y}
$$

gegeben.

\section{Bestimmung der freien Oberfläche}

Zur Bestimmung der freien Oberfläche findet die von Harlow und Welch in [1] eingeführte MAC-Methode Verwendung. Bei der MAC-Methode werden masslose Partikel zur Markierung der Zellen des Diskretisierungsgitters genutzt, die mit dem Fluid gefüllt sind. D.h., jede Zelle des Diskretisierungsgitters, die mindestens ein massloses Partikel enthält, ist Teil des Bereichs, der mit Fluid gefüllt ist. Vor diesem Hintergrund werden die masselosen Partikel als Marker bezeichnet. Wenn eine oder mehrere leere Zellen des Diskretisierungsgitters an eine mit Fluid gefüllte Zelle angrenzen, durchläuft die freie Oberfläche die Diskretisierungszelle. Ein beispielhafte Konfiguration findet sich in Abb. 3. Entsprechend Abb. 3 sind die obere linke, die obere rechte und die untere rechte Zelle leer, während die untere linke Zelle mit Fluid gefüllt ist. Folglich durchläuft die freie Oberfläche, die in Abb. 3 als gepunktete Linie dargestellt ist, die untere linke Zelle.

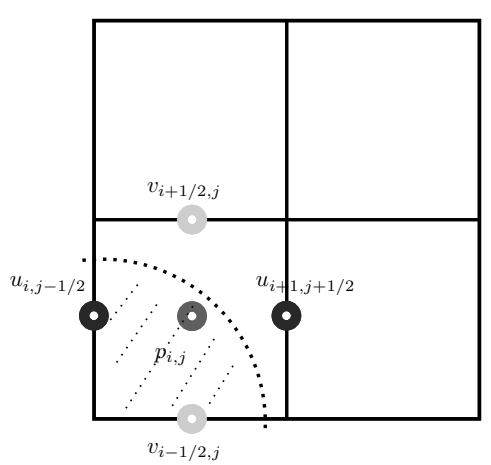

Abb. 3: Markierte Zelle mit drei freien angrenzenden Zellen

Hinsichtlich der Bestimmung der freien Oberfläche müssen die Geschwindigkeiten und der Druck berücksichtigt werden. Da bei einem inkompressiblen Fluid die Normal- und Tangentialspannungen an einer freien Oberfläche gleich Null sind (vgl. [10, 11]), müssen die Randwerte der Geschwindigkeiten und des Drucks auf 
der freien Oberfläche den Gleichungen

$$
\begin{gathered}
\frac{p}{\rho}=2 \frac{\eta}{\rho}\left[n_{x} n_{x} \frac{\partial u}{\partial d x}+n_{x} n_{y}\left(\frac{\partial u}{\partial y}+\frac{\partial v}{\partial x}\right)+n_{y} n_{y} \frac{\partial v}{\partial y}\right] \\
{\left[2 n_{x} m_{x} \frac{\partial u}{\partial x}+2 n_{y} m_{y} \frac{\partial v}{\partial y}\right]=} \\
-\left(n_{x} m_{y}+n_{y} m_{x}\right)\left(\frac{\partial u}{\partial y}+\frac{\partial v}{\partial x}\right)
\end{gathered}
$$

genügen, wobei $\mathbf{n}=\left(n_{x}, n_{y}\right)$ den Normalenvektor und $\mathbf{m}=\left(m_{x}, m_{y}\right)=\left(n_{y},-n_{x}\right)$ den Tangentialvektor darstellt.

\section{Berechnungsschema}

Schnelles Teilmodell. Die Berechnung des schnellen Teilmodells (26)-(27) erfolgt unter Nutzung der Projektionsmethode von Chorin, wobei die umgesetzte Implementierung dem in [12] vorgestellten Algorithmus folgt. Man beachte, dass $T^{k}=T^{k-1}$ für alle Integrationszeitpunkte gilt. Der Algorithmus umfasst die folgenden Schritte:

- Zeitdiskretisierung von (26)-(27) ergibt

$$
\begin{aligned}
\frac{\mathbf{u}^{k}-\mathbf{u}^{k-1}}{\Delta t} & =K\left(\mathbf{u}^{k-1}\right) \mathbf{u}^{k-1}-B p^{k}+\mathbf{f}^{k} \\
0 & =B^{T} \mathbf{u}^{k}
\end{aligned}
$$

mit der Integrationsschrittweite $\Delta t$ und dem aktuellen Integrationszeitpunkt $k$.

- Entkopplung von Druck und Geschwindigkeiten in der Impulsgleichung (31) ermöglicht das Bestimmen der Pseudogeschwindigkeiten $\tilde{\mathbf{u}}$ durch Lösen des Gleichungssystems

$$
\frac{\tilde{\mathbf{u}}-\mathbf{u}^{k-1}}{\Delta t}=K\left(\mathbf{u}^{k-1}\right) \mathbf{u}^{k-1}+\mathbf{f}^{k} .
$$

- Der Druck wird durch Lösung von

$$
\Delta t B^{T} B p^{k}=B^{T} \tilde{\mathbf{u}}
$$

bestimmt und zur Korrektur der Geschwindigkeiten gemäß

$$
\mathbf{u}^{k}=\tilde{\mathbf{u}}-\Delta t B p^{k}
$$

genutzt.
Langsames Teilmodell. Die Berechnung des langsamen Teilsystems (28)-(30) umfasst die folgenden Schritte:

- Zeitdiskretisierung von (30) ergibt

$$
\frac{T^{k}-T^{k-1}}{\Delta t}=K_{T}\left(\mathbf{u}^{k-1}\right) T(k)+D\left(\mathbf{u}^{k-1}\right)+g\left(T^{k-1}\right),
$$

sodass sie Temperatur $T^{k}$ durch Lösen von (32) bestimmt werden kann.

- Entkopplung von Druck und Geschwindigkeiten ermöglicht das Bestimmen der Pseudogeschwindigkeiten $\tilde{\mathbf{u}}$ durch Lösen der stationären, diskretisierten Navier-Stokes Gleichungen

$$
\mathbf{0}=K(\tilde{\mathbf{u}}) \tilde{\mathbf{u}}+\mathbf{f}^{k}
$$

- Bestimmung des Drucks $p^{k}$ durch Lösen von

$$
\Delta t B^{T} B p^{k}=B^{T} \tilde{\mathbf{u}} .
$$

- Korrektur der Geschwindigkeiten gemäß

$$
\mathbf{u}^{k}=\tilde{\mathbf{u}}-\Delta t B p^{k} .
$$

Bemerkung: Die Bedingungen, wann von einem Teilmodell zum anderen gewechselt werden muss, sind im Abschnitt 2.3 beschrieben.

\section{Simulationsergebnisse}

Obgleich der komplette zeitliche Verlauf der Geschwindigkeiten, des Drucks und der Temperatur berechnet wurde, werden aus Platzgründen im Folgenden nur die Temperatur und die Geschwindigkeit in $x$-Richtung zum Zeitpunkt des Erreichens des stationären Zustands dargestellt. Man beachte, dass die Berechnungen bis Erreichen des stationären Zustands ca. 10h bei Verwendung des vollständigen Modells (s. [5]) und ca. 10min bei Verwendung des reduzierten Modells dauern.

Abb. 5 zeigt das mit Hilfe des vollständigen Modells berechnete Temperaturfeld des Fluids in einem Querschnitt, der sich in der Mitte der Zylinder befindet ${ }^{1}$. Im Gegensatz dazu ist das mit dem reduzierten Modell berechnete Temperaturfeld in Abb. 6 dargestellt.

\footnotetext{
${ }^{1}$ Diese Querschnittsfläche wird in den Abb. 4-8 einfachheitshalber als mittlere Querschnittsfläche bezeichnet.
} 


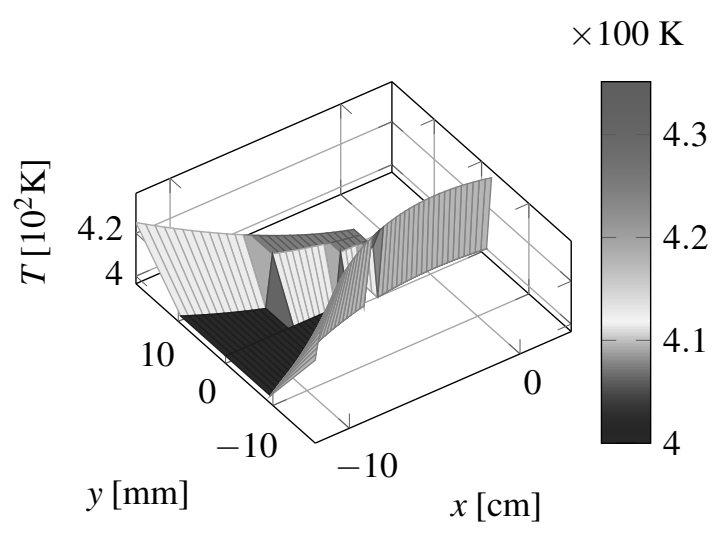

Abb. 4: Initiales Temperaturfeld in der mittleren Querschnittsfläche

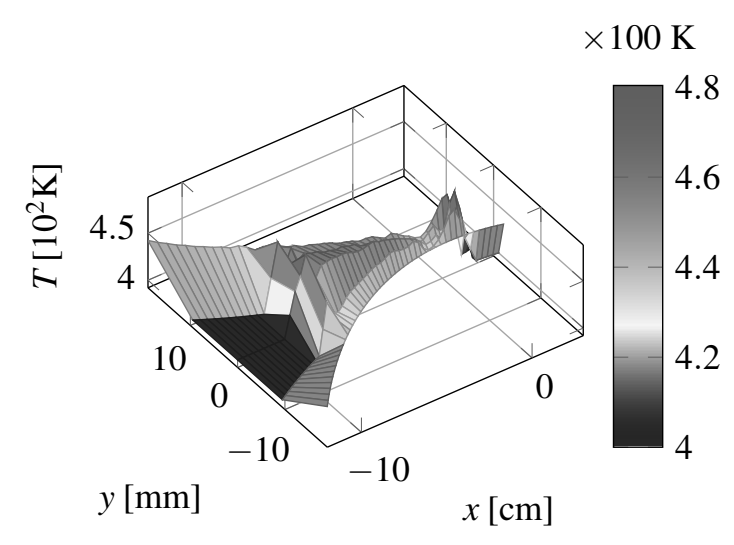

Abb. 5: Temperaturfeld in der mittleren Querschnittsfläche (vollständiges Modell)

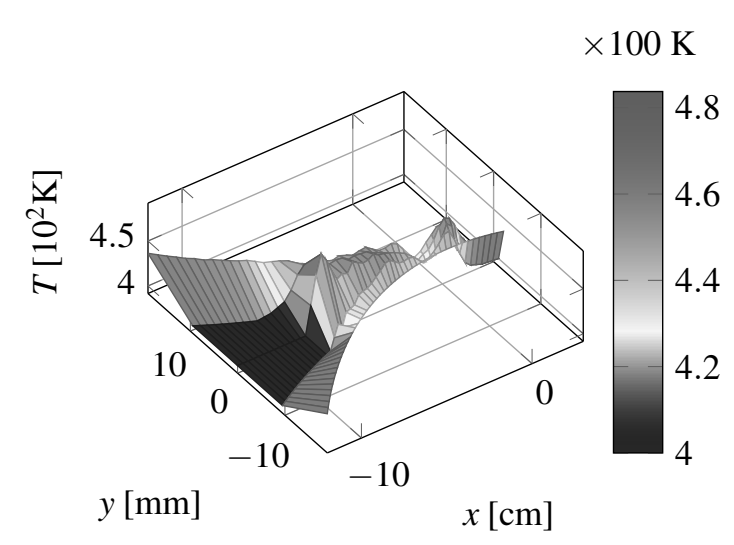

Abb. 6: Temperaturfeld in der mittleren Querschnittsfläche (reduziertes Modell)
Gemäß Abb. 4 ist die Anfangstemperatur des Fluids $425 \mathrm{~K}$, während die Umgebungstemperatur $400 \mathrm{~K}$ und die Temperatur der Zylinder $433 \mathrm{~K}$ beträgt. Obwohl es Unterschiede zwischen dem mit dem Vollmodell (Abb. 5) und dem mit dem reduzierten Modell (Abb. 6) berechneten Temperaturfeld gibt, zeigt das reduzierte Modell ein qualitativ und quantitativ ähnliches Verhalten wie das Vollmodell. Vergleicht man ferner das initiale Temperaturfeld wahlweise mit Abb. 5 oder 6, lässt sich anhand der Temperaturänderung erkennen, welchen Raum das Fluid im stationären Zustand eingenommen hat.

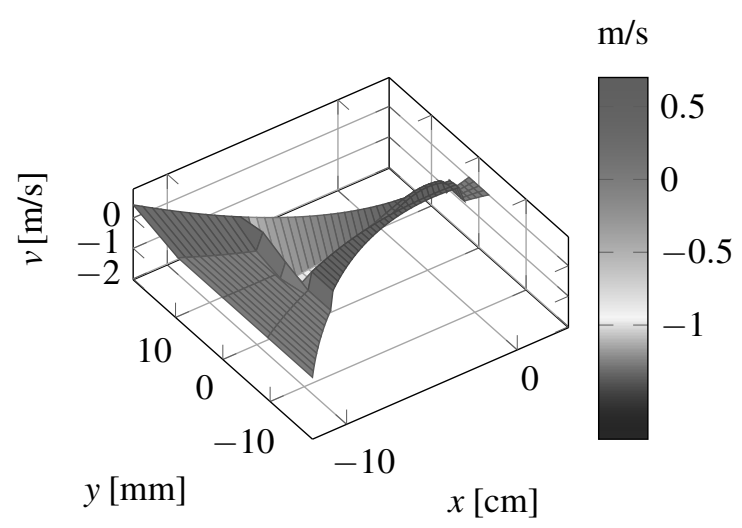

Abb. 7: Geschwindigkeitsfeld in $x$-Richtung in der mittleren Querschnittsfläche (vollständiges Modell)

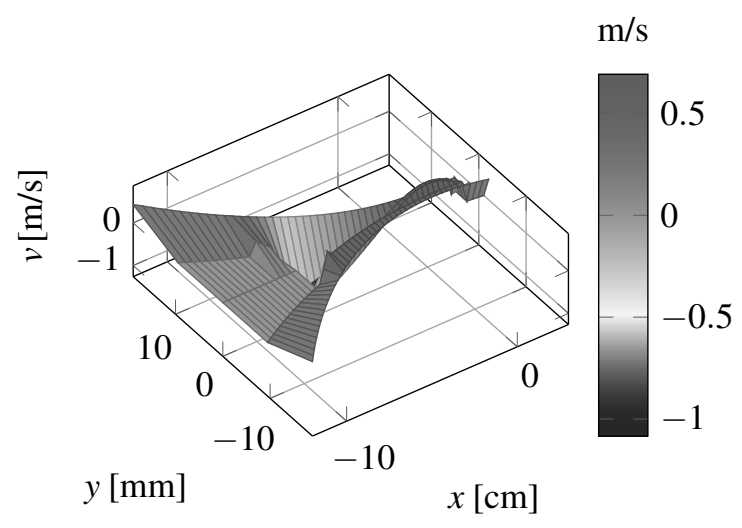

Abb. 8: Geschwindigkeitsfeld in $x$-Richtung in der mittleren Querschnittsfläche (reduziertes Modell)

Wie beim Temperaturfeld gibt es für die Geschwindigkeiten in der $x$-Richtung nur kleine Unterschiede zwischen den Ergebnissen des Vollmodells (Abb. 7) 
und den mit dem reduzierten Modell (Abb. 8) berechneten Ergebnissen. Zu beachten ist, dass im Zylinderspalt negative Geschwindigkeitswerte auftreten. Die negativen Geschwindigkeiten ergeben sich aus dem hohen Druckgradienten, der beim Eintritt des Fluids in den Spalt entsteht, sodass der Druckgradient der Strömung entgegenwirkt, was zu einer Rückströmung das Fluid führt. Dies resultiert in der bereits eingangs beschriebenen Wulstbildung. In einigen Spaltabschnitten nimmt ferner der Druck kontinuierlich ab. In diesen Abschnitten wirkt der Druckgradient in positiver $x$-Richtung, was zu erhöhten Geschwindigkeiten führt, wie den Abb. 7 und 8 entnommen werden kann.

\section{Zusammenfassung}

Die Simulation von Modellen, die das Verhalten von hochviskosen, nicht-isothermen Fluiden beschreiben, ist in der Regel mit einem hohen Rechenaufwand verbunden. Daher finden derartige Modelle weder für modellbasierte Regelungs- und Steuerungsverfahren noch für die modellbasierte Diagnose Verwendung. Mit Hilfe der Störungstheorie kann jedoch gezeigt werden, dass sich die Geschwindigkeiten und die Temperatur des Fluids auf unterschiedlichen Zeitskalen entwickeln, was darauf hindeutet, dass zwei reduzierte Modelle, d.h. ein schnelles und ein langsames Teilmodell, verwendet werden können. Mit Hilfe des schnellen Teilmodells erfolgt unter Annahme einer konstanten Fluidtemperatur, welche sich auf der langsamen Zeitskala entwickelt, die Berechnung der Fluidgeschwindigkeiten. Im Gegensatz zum schnellen Teilmodell berechnet das langsame Teilmodell die Temperatur unter der Annahme von stationären Werten für die Geschwindigkeiten, die sich auf der schnelleren Zeitskala entwickeln. Ein Vergleich der mit den verschiedenen Modellen (vollständiges Modell vs. reduziertes Modell) berechneten Ergebnisse zeigt eine hohe Übereinstimmung. Obwohl der Unterschied im Rechenaufwand sehr groß ist (ca. 10 Stunden für das vollständige Modell, ca. 10 Minuten für das reduzierte Modell), ist eine weitere Modellreduktion erforderlich, um eine sinnvolle Nutzung des Modells zur Steuerung und Diagnose zu ermöglichen.

\section{Danksagung}

Die Autoren danken der EU und dem Land NRW für die finanzielle Förderung.

\section{Literatur}

[1] Harlow, F. H., Welch J. E., Numerical calculation of time-dependent viscous incompressible flow of fluid with free surface, The Physics of Fluids, vol. 8, no. 12, pp. 2182-2189, 1965.

[2] Amsden, A. A., Harlow, F. H., The smac method: A numerical technique for calculation incompressible fluid flows, Los Alamos Scientific Laboratory, University of California, Tech. Rep., 1970.

[3] Tome, M., Filho, A., Cuminato, J. A., Mangiavacchi, N., Mc-Kee, S., Gensmac3d: a numerical method for solving unsteady three-dimensional free surface flows, International Journal for Numerical Methods in Fluids, vol. 37, no. 7, pp. 747-796, 2001.

[4] McKee, S., Tome, M., Ferreira, V., Cuminato, J., Castelo, A., Sousa, F., Mangiavacchi, N., The mac method, Computer \& Fluids, vol. 37, no. 8, pp. 907-930, 2008.

[5] Harder, D., Skeli, E., Weidemann, D., Modelling and simulation of high-viscosity, non iso-thermal fluids with a free surface, in Proc. of the 15th Conference on Informatics, Automation, and Robotics (ICINCO), pp. 557-563, 2018.

[6] Lichte, B., Verläßliche und effiziente Simulation physikalisch-technischer Systeme durch Nutzung von Fachwissen, Shaker Verlag, 2006.

[7] Panreck, K., Verkopplungsorientierte Modellbildung und Simulation instationärer Extrusionsprozesse, Dissertation, Univ. Paderborn, 1995.

[8] Kokotovic, P. V., Singular perturbation techniques in control theory, in Singular Perturbations and Asymptotic Analysis in Control Systems, Kokotovic, P. V., Bensoussan, A., Blankenship, G. L. (Editoren), pp. 1-55, Springer, 1987.

[9] Verhulst, F., Methods and applications of singular perturbations - Boundary layers and multiple timescale dynamics, Springer, 2005.

[10] Hirt, C. W., Shannon, J. P., Free-surface stress conditions for incompressible-flow calculations, Journal of Computational Physics, vol. 2, no. 4, pp. 403-411, 1968.

[11] Nichols, B. D., Hirt, C. W., Improved free surface boundary conditions for numerical incompressible-flow calculations, Journal of Computational Physics, vol. 8, no. 3, pp. 434-448, 1971.

[12] Weickert, J., Applications of the theory of differential-algebraic equations to partial differential equations of fluid dynamics, Dissertation, TU Chemnitz, 1997. 